\title{
FROM POINT CLOUD TO 3D MODEL, MODELLING METHODS BASED ON ARCHITECTURAL KNOWLEDGE APPLIED TO FORTRESS OF CHÂTEL-SUR-MOSELLE (FRANCE)
}

\author{
E. Alby, P. Grussenmeyer,
}

The Image Sciences, Computer Sciences and Remote Sensing Laboratory

LSIIT-TRIO UMR 7005, INSA Strasbourg, France

Corresponding author: Emmanuel.alby@insa-strasbourg.fr

\begin{abstract}
KEY WORDS: Cultural Heritage, Method, Simplification, Representation, Modelling
\end{abstract}
\begin{abstract}
:
3D survey techniques applied to buildings such as photogrammetry and laser scanning produces a volume of data very important. Upon acquisition, the issue of data management greatly influences the acquisition parameters and production data. Contemporary tools usually allow acquiring data sets denser than necessary without taking a very long acquisition times. We are interested in modeling from 3D data. There is no question here of mesh from the point cloud, but to model space according to their constituent elements. Data having a high density provide a rich description of the object. The issue of simplification raises the problem of loss of means of describing the object of interest. The final model, to keep the quality of description of point clouds, must remain very close to the initial data. This constraint derives the inverse problem. It is to be faithful to the data without performing mesh. The purpose of the study must remain the goal of modeling. Assumptions used to derive simple geometric primitives, which are assembled together the simplified model. The resulting model consists of surface distance for $92 \%$ of them within $5 \mathrm{~cm}$ of the point cloud. The model thus created is faithful to the cloud but also the rules of construction of the building which he prefigures representation.
\end{abstract}

\section{INTRODUCTION}

The acquisition by laser scanning has become very fast Furthermore, the setup time becomes incompressible when you consider the total duration of a station. A time-of-flight scanner is very fast; a phase-shift device scans almost instantly. What is the influence of the density of points needed on the duration of the scan? It is less and less significant. The volume of data is increasing constantly. The first treatment is more often the arbitrary reduction of the amount of point so that the workstation can open the cloud. The amount of data has become impressive when you consider the raw data. How is the transition from data to representation during treatments? The mesh applied to the point cloud is often a way to obtain a surface representation. If there is a texture captured with the cloud that is associated to the mesh, the resulting model becomes very difficult to display. If the mesh is a way to faithfully represent an object [1], what are the maximum dimensions of an object, if it has to be meshed and correctly displayed? Fortress of Chatel sur Moselle is a remnant of an impressive defensive site of the middle ages. The interior galleries spread over three levels are being documented in 3D by laser scanning [2]. The cloud, even limited to a distance of one centimeter between points, cannot be processed into a mesh and be easily manipulated. The model should serve as a communication medium on the one hand and a tool for archaeologists. It must be easily manipulated. This defensive structure without decoration is a good example to process simplification to reduce the amount of data. It was build according to traditional methods of construction and allows making the assumption of use of geometric primitives that will be presented in this paper. The method developed for this project is explained. At last the results are presented.

\section{MODELING WITH GEOMETRIC PRIMITIVES}

\subsection{D model as representation}

The three-dimensional model is primarily a representation. It is a way to communicate the shapes of a building by a direct analogy to its dimensions [3]. The representation of an object and more particularly of an architectural space is subject to specific constraints. The plane representation of the architecture is the result of a long process of codification. The plane representation gives the possibility to spend from a threedimensional object to a two-dimensional description with minimal loss of information [4]. When there is a plan representation, representation corresponds to a defined scale. Once the representation scale of the object is defined, measurement accuracy is defined. Regarding the 3D model, there are fewer rules. The precision of the acquisition and the density of points, are less subject to codification that for the representation in two dimensions. These parameters are more depending of a precise specification before the production of each model. The 3D display is not limited to the size of the screen. It is even less restricted to a paper size. The ability to zoom alters the perception of object representation. Is it thinkable to display everything because this has become possible?

The boundary between relevant representation and willingness to show more precisely is even easier to cross for a $3 \mathrm{~d}$ model thanks to the editing capabilities offered by computers.

\subsection{D model easy to manipulate}

The potential of computer science are ever increasing. With regard to our subject: displaying a 3D model, the best workstations have their own limitations [1]. We addressed the question of the relevance of the representation in the previous paragraph.

The ability of a 3D model to be manipulated is a strong constraint that it is imperative to respect [5]. The model loses its interest when it is not easily visualized. Considered as a communication tool for the general public or as an analysis tool by archaeologists, the $3 \mathrm{D}$ model must be built with the same care for display efficiency. If the user cannot make the most of 
the representation made to him, the objective of the $3 \mathrm{D}$ model is not reached. Under these conditions, the result he offers is only partially achieved.

\subsection{Compromise between fidelity to the dimensions and exploitation}

Considering ease of manipulation previously described as essential, it remains to consider the main objective, which is the motivation of the representation of 3D model. In the community of $3 \mathrm{D}$ acquisition, the dimensional analogy between the real object and the 3D model is the dominant factor.

The 3D model must be faithful to reality through a direct relation to the dimensional characteristics of the object described. Whether to produce a 3D model that satisfies these two constraints are: fidelity to the object and ease of manipulation, we must find the best compromise between these two parameters that can be contradictory. The surface representation that is most faithful to the raw data represented here by the point cloud is the mesh. A fine mesh provides a very rich rendering of the modeled object. If we mesh a point cloud of the same object that has been resampled with a lower density, the resulting mesh loses this render quality made to move towards an aberrant representation [6]. If the mesh provides a efficient description when it is produced from a dense point cloud, when the density of the cloud decreases, the relevance decreases.

\subsection{Surface model based on geometric primitives}

The mesh is a surface model produced automatically. If it is simplified, the 3D model generated loses its relevance. If you want to reduce the amount of data to display, while limiting the loss of relevance of the representation, the surface representing the object must come from another method. It is interesting to consider how the built works were carried out to make an analogy between implementation and construction of the 3D model of the same building [7]. The builder of ancient buildings, that have used only drawing tools based on the line and arc, have produced buildings largely decomposed by this drawing primitives. This is the hypothesis we make in this study, to produce the surface that will describe the object of our study, in the proposed model.

\subsection{Adaptation of geometric primitives with the constructive principles}

The model resulting from this study will be constructed from geometric primitives

It is easy enough to understand that a flat wall is easier to build than a wall made from another form. Its realization will be more easily controlled. Furthermore a vertical load is easier to transmit with a vertical wall [8]. It is with these same arguments that we arrive at the representation of vaults by portions of cylinders and cones. The forms of vaults must be drawn before being build, thus found in the greatest number of cases, arcs as the vaults generators. Deformations due to time damage can disturb this logic, one must carefully control the primitives used to remain faithful to the original building.

\subsection{Hypothesis facilitated by the lack of decoration}

The work we present in this study is a defensive structure. This fortress, as a military building consists of volumes rather simple and very few decorated. There are no elements of moldings. They also have no influence for the generation of the shapes of the spaces. Furthermore, the existing state of the building is the result of many restorations, which minimizes the walls distorted by time.

\section{METHOD}

Unlike the meshing process, which has the great advantage of being highly automated, the method proposed in this study requires more operator intervention. Unlike the mesh that must still be supervised, controlled and edited at the end of the build process, our method requires manual operations early in the process.

\subsection{Determining the largest adjusted primitive}

To minimize the model, it is necessary that the primitives chosen to represent properly the subject are as large as possible. The objective is achieved gradually by looking for discontinuities in the surfaces.

\subsection{Correspond to the point cloud with the closest primitive}

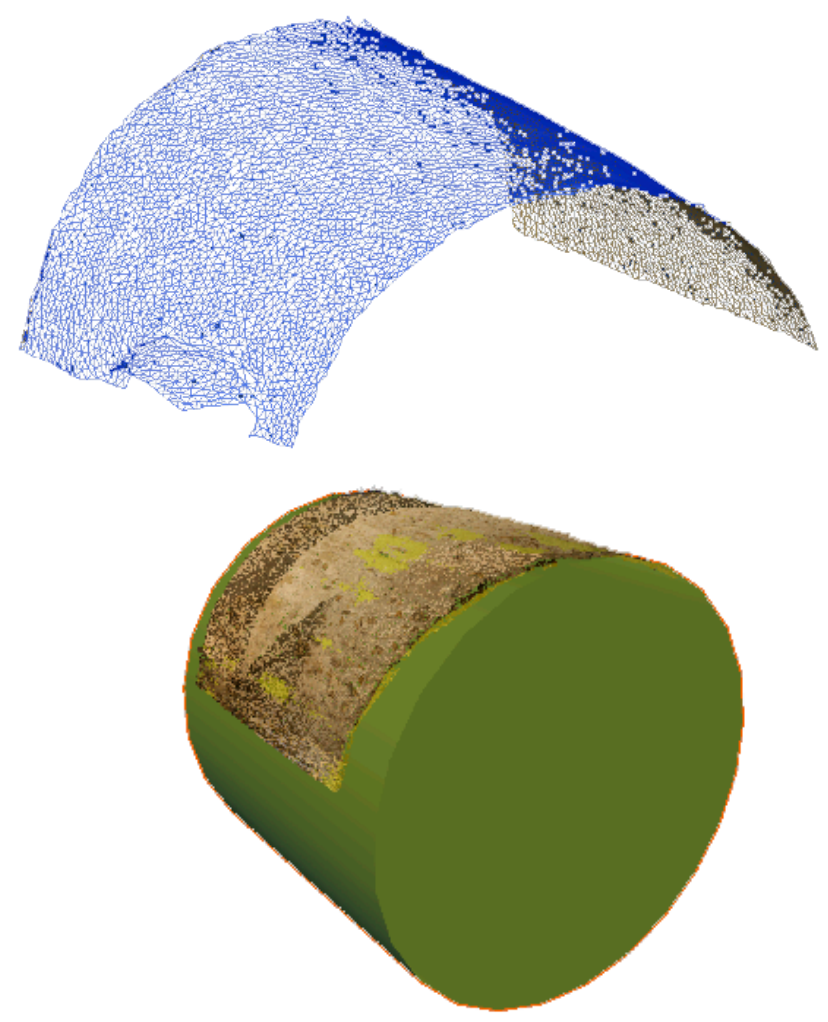

Figure 1: Definition of the primitive for a vault. Up, a mesh from point cloud; down, detection of the cone closest to the mesh.

The primitive shall be adjusted as closely as possible to the point cloud. It can match the most plausible representation of the building. It must simultaneously be as close as possible to the data. The result must therefore correspond to the building from the point of a view of the geometric shape and from the point of view of its dimensional characteristics. Once the choice of the corresponding type of primitive is made, this choice must be validated by the adjustment of the primitive to the cloud as shown in Figure 1. Depending on the shape of the object, the 
solutions proposed by the algorithm can be better adjusted to the cloud point without correspond to the shape of the object. Figure 2 shows two primitives from the same cloud, one corresponds to the cloud, the other is an aberration. Il must then be controlled. Depending on the initial deformation of the surface, the primitive can be adjusted with aberrant generators. For a vault that looks cylindrical, it may be necessary to choose a cone if the radius varies. Control of the outcome provides the most accurate representation.

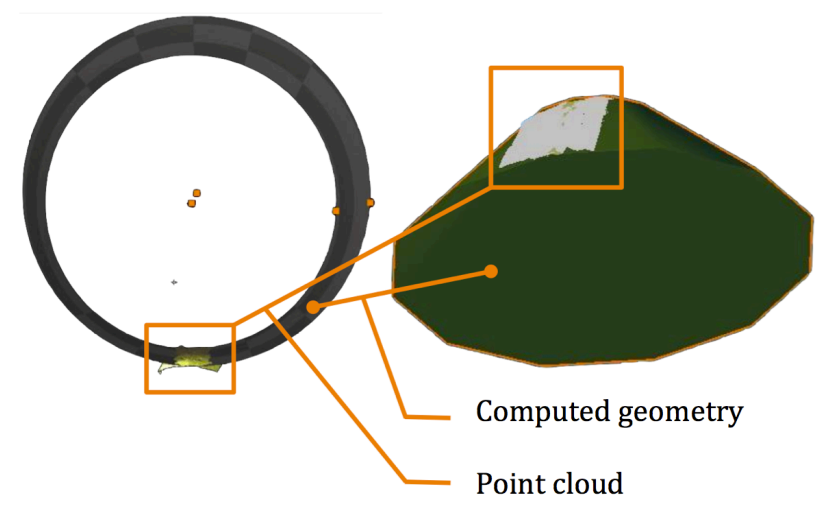

Figure 2: geometric primitives derived from the same cloud point. On the right the shape is aberrant regarding to the cloud, while it correctly matches to the point cloud on the left.

\subsection{Repeat the process for the whole point cloud}

The preceding paragraph describes the modeling process for matching the object with a single geometric primitive. This step corresponds to only a very limited portion of the object. The model we want to achieve is the interior of the fortress of Châtel sur Moselle. It consists of ground elements, walls, vaults, vast spaces and windows. The spaces are large when they are rooms with specific functions, such as the guardroom for example, or close when it comes to stairs or release. The size of spaces does not necessarily affect the number of primitives to be used for modeling. A narrow staircase requires a lot of primitives because of the steps, for example, while a room of rectangular plan is fairly easy to model relative to its size.

The stage of the previous chapter will therefore be repeated at least a dozen times or more for each space of the model. The connections between the different primitives must be carefully made. As precised for the generators of a cone in the case of a vault, the intersections between different plans must follow the right directions considering the initial space. For an access gallery to a window that follows different angular directions ; the resulting edges from the intersections of plane walls should be as vertical as possible.

\subsection{Cleaning the resulting model}

The primitives adjustment takes place in a software of point cloud management and postprocessing like RealWorks or Cyclone. All primitives must be intersected with each other properly. The software mentioned above contain these intersections management tools, but it does it only correctly between pairs of primitive. The problem of modeling proposed in this study, even if based on accurate data type like point cloud is like 3D modeling in computer graphics.

Using a 3D modeling software is most appropriate here. The transition from one program to another must be made properly in order to preserve all the qualities of model accuracy. The point cloud is georeferenced, and the town of Chatel sur
Moselle is located east of France, the coordinates are composed of nine significant digits ( 6 for the integer part and at least 3 for decimals).

the 3D modeling softwares is generally designed to work with the origine of the frame near the object. the $3 \mathrm{D}$ modeling softwares are not designed for managing coordinates so high and sometimes when importing, coordinates are truncated to 0.1 or 0.01 . To avoid this, the solution is to translate the data to increase accuracy controlled by software and put the origin of the frame near the object.

The intersection and / or extension of the primitives can be made.

\subsection{Comparison with the point cloud}
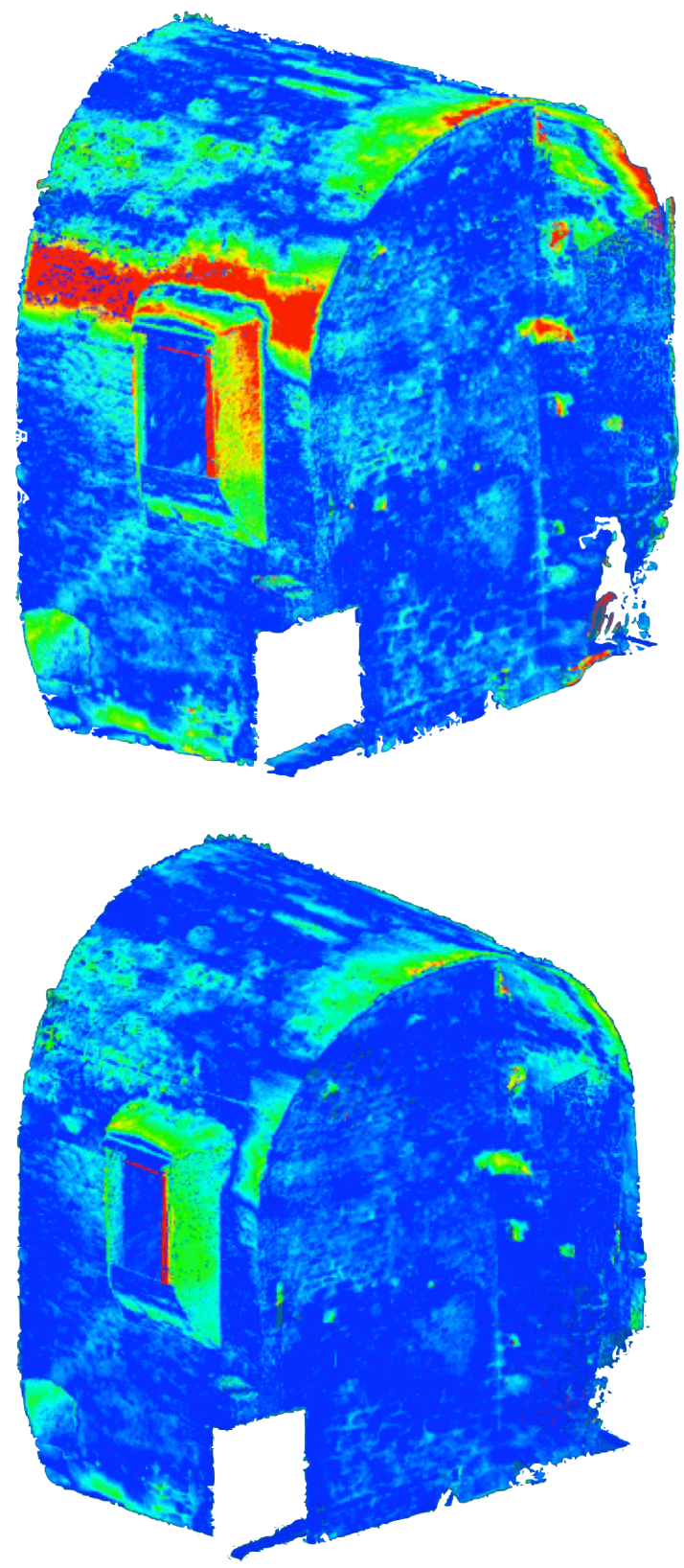

Figure 3. Improving the model by comparing the point cloud; on upper picture, the area in red shows wrong choices corrected on the picture below. 
The model is close to its final version, where everything has been cleaned and adjusted. The problem of our study is linked to a respect for accuracy of original $3 \mathrm{D}$ data; it is essential to check whether the simplifying assumptions generalized to the whole object are valid. The simplified model should be as close as possible to the point cloud. The resulting model should be compared to the origin point cloud. Editing software of clouds and meshes allow such inspections. The result coded by color scale is used to verify the accuracy of the model and identify areas for improvement as shown in Figure 3. Analysis of the results should get in touch with the density of the cloud of points, the measurement accuracy of laser scanner and the accuracy of implementation for buildings of this type.

\subsection{Improvement areas outside tolerance}

The full model refers directly to the original data. It is compared to the point cloud to find the areas that must be corrected. Modifications must be realized by finding the best fit of primitive causing the error. If any good result is obtained, thus another primitive must be used (instead of cylinder cone for example). Once the adjustments corrected, the primitives used should be put back into the model, to fit over adjacent primitives. If the comparison of the model with the cloud still requires corrections, repeat these steps again.

\section{RESULTS}

This study is motivated by the desire to obtain a model that is lightweight, to facilitate its handling and both faithful to the original data.

\subsection{Lightweight model, easy to handle}

To evaluate the optimized model, we compare the number of triangles in the model and the corresponding mesh for a given room.

Both types of surface models are converted in the same format (here *.Obj) and edited in the same software (3DReshaper here) for a similar account of triangles. The initial point cloud consists of 2.2 million points. The meshing of point cloud contains about 100000 triangles. It has not been made between all points; basic simplifications were made. The model based on primitive contains about 2000 triangles. Figure 4 shows the point cloud of the guardroom, the mesh model and the corresponding simplified model. The primitive model-based geometric contains 50 times fewer triangles than the mesh from point cloud, as shown in Table 1. The model produced is much more manageable in terms of $3 \mathrm{D}$ performance of the computer that will display it.

\begin{tabular}{|c|c|}
\hline & Number of entities \\
\hline Point cloud & $2,200,000$ points \\
\hline Meshed model & 100,000 Triangles \\
\hline Simplified model & 2,000 Triangles \\
\hline
\end{tabular}

Table 1: summary of amounts of data for the object Guard Room a)

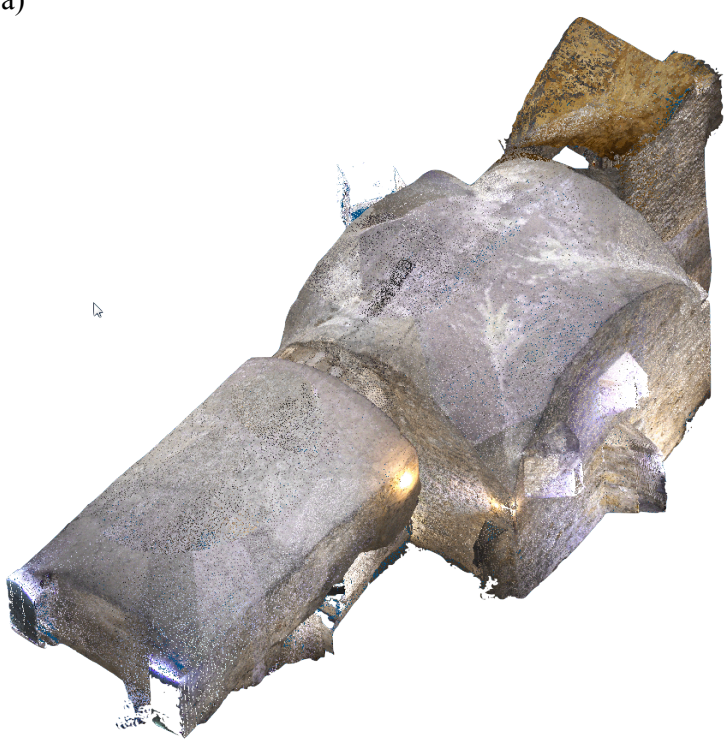

b)

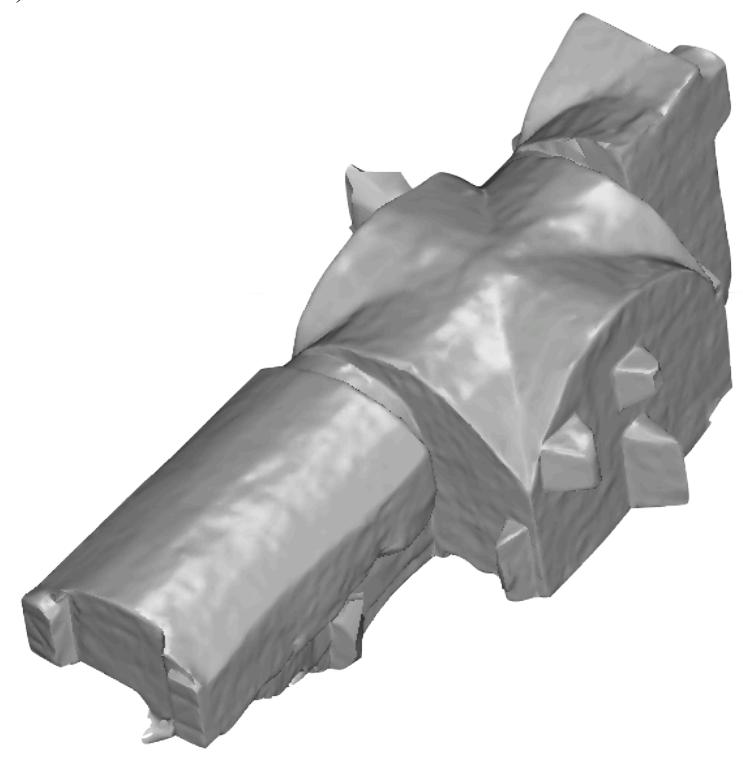

c)

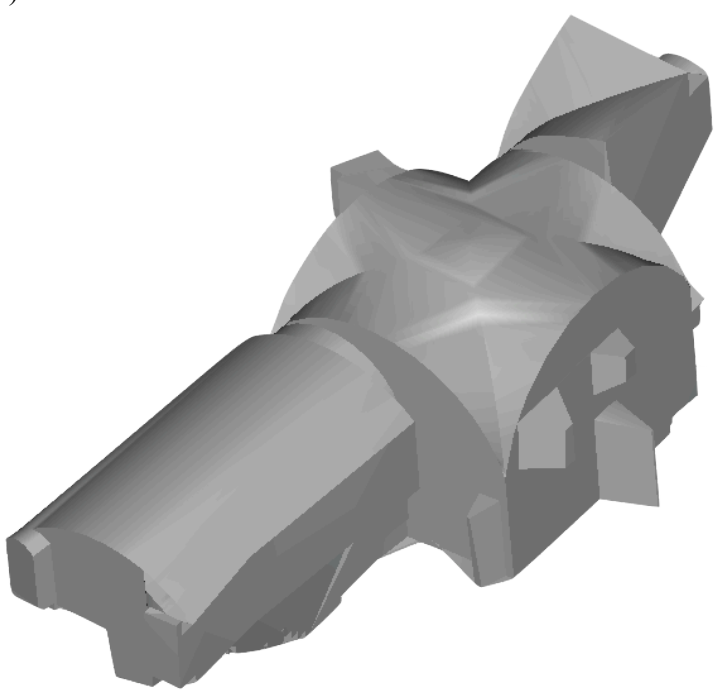

Figure 4. Guard room, a) Point cloud, density $1 \mathrm{~cm}$; b) mesh applied to point cloud; c) Simplified model. 


\subsection{D model very close to the cloud}

The concern of fidelity to point cloud that was present throughout the development of the model yields the following result: $92 \%$ of faces are within $5 \mathrm{~cm}$ of the cloud. Knowing that the walls are not plastered, some blocks are not aligned with the wall, and holes that remain after the fall of blocks, we can consider this result as satisfactory. Figure 5. Shows the comparison result of the simplified model with the cloud point of the entire area modeled in 2010.

\subsection{Easy way to continue}

The study presented here is at the start of a long survey of the inner parts of the fortress of Châtel-sur-Moselle. One constraint was to provide a method allowing a person to pursue modeling and get the same results consistent with the existing model and adjusted to the point cloud. Despite the different crossings between software followed by return to edits the process allows a continuity of building the model of independent operators.

a)

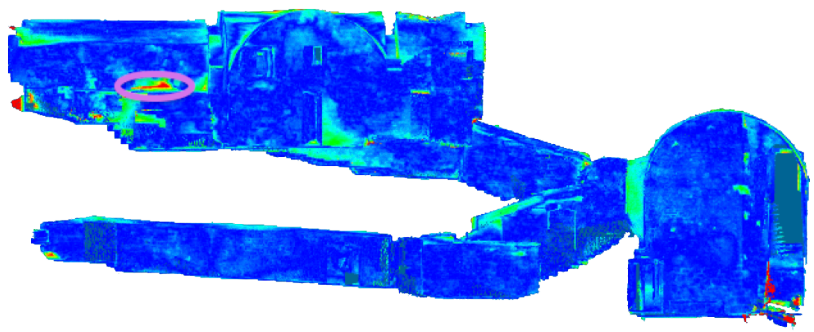

b)

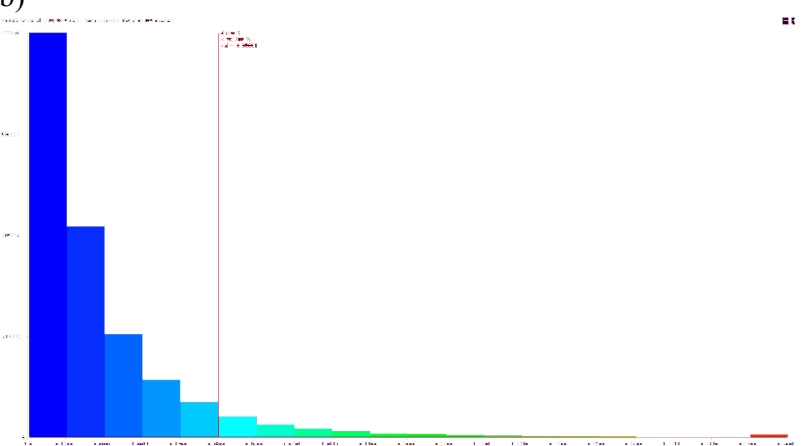

Figure 5. a) Result of the comparison faces / cloud; b) $92 \%$ of distances below $5 \mathrm{~cm}$.

\section{CONCLUSION}

When a $3 \mathrm{D}$ model have to be presented to the public eye, the optimization is essential. Rendering performance is often at the expense of fidelity to the original. The model of the inner parts of the fortress of Chatel sur Moselle is intended for two contradictory purposes. The first is to show the model to a very large number of people. The second to serve as support work for archaeologists. Easy viewing and accuracy are not additional parameters, but they were both taken into account and that's what led to our study. The results show that the two constraints are not necessarily contradictory, as long as the means of implementation of the builders of the ancient time are taken into account and that the operator reclaims them to build the model. The very precise data of departure are sidelined, initially, the better to integrate them at the end. If the results are interesting at first sight, the need for automation of our method has to be taken into account. The geometric model can be associated with the textures of moderate weight to keep the facility of visualization and allow visitors to get caught by the magic of the place. Figure 6 gives an overview of the textured model.

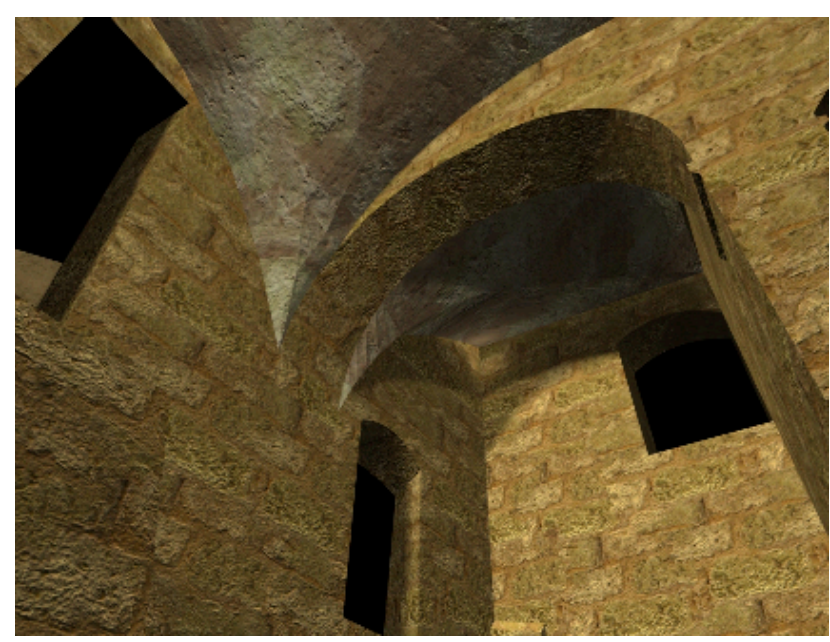

Figure 6. overview of the textured model.

\section{ACKNOWLEDGEMENT:}

It is important to thank Valentin Poitevin for his enthusiasm and really works, which has experimented this method on the first data from the field. The devices do not provide the best data sets without the right parameters and tenacity of Samuel Guillemin, who handles it.

\section{BIBLIOGRAPHY}

References from Journals:

[1] Remondino, F; El-Hakim, S. Image-based 3D modelling: A review. Photogrammetric Record 2006, 21, 269-291

[2] Alby, E., Poitevin,V., Grussenmeyer, P, 2011. From 3D recording to virtual visit of archaeological sites: methodology applied to the medieval fortress of Châtel sur Moselle (France). CIPA International Archives for Documentation of Cultural Heritage, XXIII, 6 p., (2011)

[3] Sven Havemann, Dieter Fellner, A versatile 3D model representation for cultural reconstruction, Proceedings of the 2001 conference on Virtual reality, archeology, and cultural heritage, November 28-30, 2001, Glyfada, Greece

[6] Luebke, D., 2001. A developer's survey of polygonal simplification algorithms. IEEE Computer Graphics and Applications, 21(3): 24-35.

[5] Grussenmeyer, P., Alby, E., Meyer, R., Rampazzo, M., 2006. 3D building model as an interface for a Web Information System. Case study of the Pontonniers high school in Strasbourg. ISPRS Comm. V Symposium, Dresden, Sept. 2527, Germany. Int. Arch. of Photogrammetry and Remote Sensing and Spatial Information Sciences, Vol. XXXVI, Part 5, ISSN 1682-1750, 6 p.,(2006)

[7] Alby E., Grussenmeyer P., Perrin J.P., 2005. Analogy between architectural design process and the documentation of 
International Archives of the Photogrammetry, Remote Sensing and Spatial Information Sciences, Volume XXXIX-B5, 2012 XXII ISPRS Congress, 25 August - 01 September 2012, Melbourne, Australia

architectural works. CIPA International Archives for Documentation of Cultural Heritage, XX, 6 p., 2005

References from Books:

[4] Saint-Aubin J.-P., Le relevé et la représentation de l'architecture, Inventaire Général, E. L. P., 1992.

[8] Perouse de Montclos J.-M..(2011) Architecture : description et vocabulaire méthodiques, Inventaire général du patrimoine culturel ; réd. Jean-Marie Pérouse de Montclos. - Paris : Editions du patrimoine, Centre des monuments nationaux, 2011 - 672 p., édition revue et augmentée. - ISSN 0768-0031 